\title{
A METHODOLOGY FOR DEVELOPING VIDEO PROCESSING
} SYSTEM

\author{
C.Keerthana ${ }^{1}$, G.Rameshchandra ${ }^{2}$ \\ ${ }^{1}$ C.Keerthana P.G Student, Software Engineering, VNR Vignana Jyothi Institute of Engineering and Technology, \\ Telangana, India \\ ${ }^{2}$ Dr.G.Rameshchandra, Professor, Computer Science and Engineering, VNR Vignana Jyothi Institute of Engineering \\ and Technology, Telangana, India
}

\begin{abstract}
The data is exploding day by day in digital technology. Now a day's multimedia data is also handled by the database, multimedia data contains data like images, text and video. The video processing plays a tremendous role in the multimedia but all the videos are not same, it can exists number of settings and different number of formats. By This video processing system the video is processed for enhancement, analysis, dividing the channels and binarization by using different image processing techniques. In this system different color system like YCBR, HSL, and RGB color systems are considered for processing any type of video. For this system, the input video can be from a stored file or continuous stream of video sequences from the web camera (or) any type of camera by this video processing system we can improve the quality of the video and we can also apply some special effects to the video by applying various image processing techniques and filters. The enhancement techniques considered in his system are filtering with correlation and convolution, adaptive smoothing, conservative smoothing and median filtering. The analysis techniques like edge detection, histogram and statistical analysis are considered for this system. Binarization methods implemented in this system are Custom Threshold, Order Dither. The Color filters like converting RGB to Grayscale, Grayscale to $R G B$,Sepia, invert, rotate, Custom Color filter, Euclidean color filter, channel filter, red, green, blue, cyan, magenta and yellow, they are so many other filters are also implemented in this system.
\end{abstract}

Key Words: Enhancement, Analysis, Dividing the channels, Binarization

\section{INTRODUCTION}

Computer graphics and computer vision always deals with the image processing techniques but most of the image processing techniques are applied to images but not for the videos. Videos are nothing but sequence of images. The video processing has experienced a tremendous growth over past decade in multimedia but not all the videos has same formats and settings it consists of different formats and as many number of settings by this the quality of the video is missing. In present system it consists of very limited functionality and with more rendering delay. In this proposed video processing system the dynamic histogram is used and the video undergo in terms of enhancement, analysis, dividing the channels, binarization and different color systems like YCBCr, HSL and RGB color systems and different image processing techniques are used for processing any type of video to improve the quality.

(a) Enhancement: By applying the enhancement we can improve the quality of video by manipulating the picture for example, to make a picture lighter or darker, or to expand or curb contrast etc.

(b) Analysis: Hear the structure of the video is examined in detailed by applying different edge detection techniques.

(c) Binarization: Hear we can convert the gray scale document image to a binary document image.

\section{METHODOLOGY}

Coming to the methodology the input file can be from a stored file or continuous stream of video sequences from a web camera or any type of camera. The input video is divided into number of frames each frame is processed by using image processing techniques like segmentation, analysis, binarization and dividing the channels. After processing the video the result is placed in the video RAM and the processed video is displayed on the form. Again it takes another frame and repeats the process until the last frame after completing the last frame then the video get stop and exit. The following figure 1 describes the methodology of video processing system.

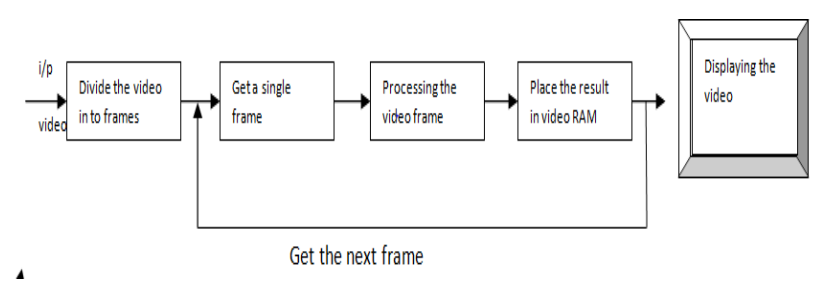

Fig -1: Methodology of video processing system 


\subsection{Processing The Video:}

The video is processed by using many image processing techniques. These image processing techniques are further classified in to two types such as operations and filters.

\subsubsection{Operations:}

There are different types of operations applied on a video frame such as Flip, Mirror, Rotate and Zoom.

(a) Flip: The Flip operation is used to rotate the image to top-to-bottom or to reverse the image to left-toright a bitmap in a memory and it displays results on the screen.

(b) Mirror: This filter mirrors image around $\mathrm{X}$ or $\mathrm{Y}$ axis.

(c) Rotate: This rotate operation is used to rotate the image in different angles

(d) Zoom: This zoom operation is used to change the size of the image. We can increase or decrease the size of the image.

\subsubsection{Filtering:}

There are different types of filtering techniques applied on a video. But we mainly discuss about three filtering techniques they are Low pass filtering, High pass filtering and Median filtering, these filters are described in a brief .

(a) Low pass filtering: Low pass filter is also known as "Smoothing" it is used to remove the high spatial frequency noise from an image. These noises are introduced when the analog image is converted in to the digital image. It generally reduces the noise and makes the image smoother. Smoothing levels are changed by increasing the window size the following figures 2 show the mask of the low pass filtering.

\begin{tabular}{|l|l|l|}
\hline $1 / 9$ & $1 / 9$ & $1 / 9$ \\
\hline $1 / 9$ & $1 / 9$ & $1 / 9$ \\
\hline $1 / 9$ & $1 / 9$ & $1 / 9$ \\
\hline
\end{tabular}

Fig-2: mask of the Low pass filter

(b) High pass filtering: High pass filtering is used to identify the edges of the image so this filter is also known as "edge detection". Edge detection is a set of mathematical methods which aim at identifying the points in a digital image at which the brightness of the image changes sharply. The points at which image brightness changes sharply are mainly equipped in to a suite of curved line segments termed as edges. The purpose of finding edges is to capture important events and properties of the image in this filters we use negative weights in the mask this results in negative values. The pixel value should be range between [0-255] in this video processing system we are applying different types of edge detection filters such as Homogeneity edge detection. The following figure 3 shows the mask of the high pass filtering.

\begin{tabular}{|l|l|l|}
\hline-1 & -1 & -1 \\
\hline-1 & 8 & -1 \\
\hline-1 & -1 & -1 \\
\hline
\end{tabular}

Fig-3: High pass filtering

(c) Median filtering: Low pass and High pass are always described by a weighting mask on the other hand Median filtering belong to a class of rank filters. Hear the pixels are ranked within the window, according to the ordering of the pixel values the result of filtering is chosen, the new pixel value will be the median of the pixel values in the window. By using this type of filtering techniques we can remove the noise of the image.

Many filters are used by applying the filtering techniques in these video processing techniques some of them are given below,

- Grayscale: By using the grayscale filter the image converts into black and white image. This filter exclusively composed the image in to gray shades. Hear the intensity of the black is weak and the intensity of the white is strong. In this type of images the intensity of light is measured at each pixel.

- Sepia: Sepia is a dark brown-grey color filter. It makes an image frame look like an old brown photo.

- Threshold: By using this filter, binarization of image is done with specified threshold value. The value of the pixels which are equal or grater than the threshold value then the pixels is changed to white pixels. Rest of all other pixels which are below than the threshold values are changed to black pixels.

- SobelEdgeDetector: Sobel filter is one of the edge detection filters. By applying this filter it first searches for the edges of the object. Hear $3 \times 3$ kernels are used to calculate the gradient magnitude for the pixel of the source image.

- Blur: The video frame is blured by applying this filter.The different types of blur filters are Gaussian blur, Simple blur, Selective blur and Pixelize.

- Invert filter: By applying the invert filter to the video it gives the effect of negative image in the video frame.

- Convolution: This filter implements convolution operator, which calculates each pixel of the result image as weighted sum of the corresponded pixel -and its neighbors in the source image. The weights are set by convolution kernel. The weighted sum is divided by Divisor before putting it into result image and also may be threshold using Threshold value.

Convolution is a simple mathematical operation which is fundamental to many common image processing filters. Depending on the type of provided kernel, the filter may produce different results, like blur image, sharpen it, find edges, etc. 
The filter accepts 8 and 16 bpp grayscale images and 24, 32, 48 and 64 bpp color images for processing. Depending on the value of Process Alpha property, the alpha channel is either copied as is or processed with the kernel.

- Magenta: Magenta is one of the color filters. By applying this filter the image is converted into bright purple pinkish color.

- Salt-and-Pepper Noise: This filter ads salt and pepper noise to the image, it sets maximum or minimum pixels values to randomly selected pixels. The image with salt and pepper presents black and white pixels spread over on the image. It accepts only $8 \mathrm{bpp}$ grayscale images and 24/32 bpp color images for processing.

- Adaptive Smoothing: Adaptive Smoothing filter is used to perform image smoothing, it gives sharp edges. It only applicable to additive noise removal, the interior part of the image is smoothening by keeping the sharp edges of an image.

- Contrast Stretch: Contrast stretch is similar to the normalization. It is one of the image enhancement techniques, the histogram stretches automatically, maximum and minimum values are used to stretch the Red, Green, Blue histograms. The brighter color appears brighter and the darker ones appear darker when contrast is increased. Contrast stretch is used to remove unwanted color tint and remains pure white and pure black in the image. This filter accepts $8 \mathrm{bpp}$ grayscale and 24 bpp color images.

- Ordered Dithering: Ordered dithering is same as Threshold filter, the pixels value is changed to white. If the intensity of pixel is higher than he threshold value then the pixels changes to white and when the intensity of pixels are lower than the threshold then the pixels changes in to black. The ordered dithering uses matrix of threshold values instead of using single threshold value. This filter accepts 8 bpp grayscale images for processing.

- Oil Painting: By applying the oil painting filter each pixel value is changed with the most frequent intensity. The frequent intensity value should be taken within the specified size of window, and the most frequent intensity value is placed at the center of the window. By this process the image will get Oil painting effect.

- Color Filters: By using color filters the image is filled with specified color inside and outside with specified range. Some of the color filters like HSL filtering, HSL linear, YCBCR filtering, YCBCR linear, YCBCR extract channel accepts only 24 and 32 bpp color images for processing.

\section{RESULTS}

Some of the screen shots are shown below when we apply different filters on the video.

3.1 Main Form: Figure 4 shows the Main Form of a methodology for developing video processing system. This is used to open the video by choosing a file in the directory. Hear we can see the menu items life file, Image, Filters, Help etc.

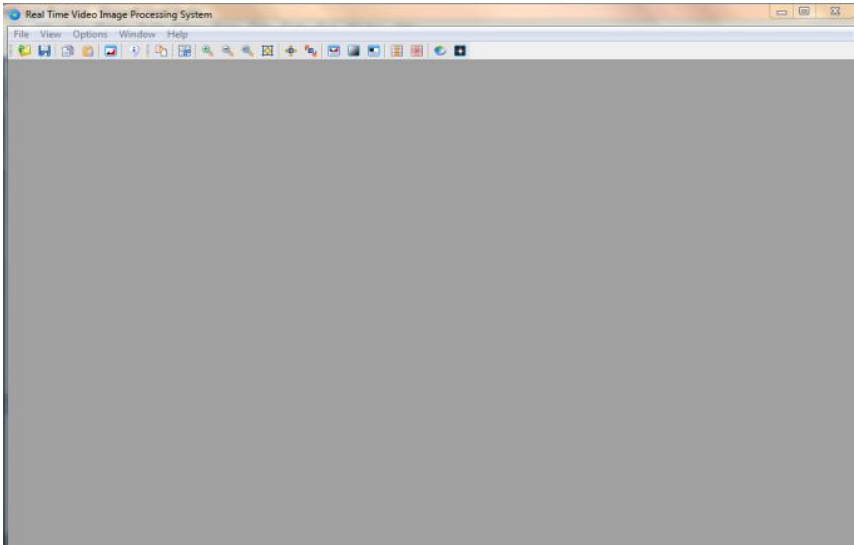

Fig-4: Main Form

3.2 Image Doc Form: Once the video is opened for processing the filter in the Image Doc form is activated. In figure 5 the Zoom operation is applied. Hear the video is Zoomed to $200 \%$ the video can be zoomed to the specifed range but the auality of the video remains same even the video is zoomed.

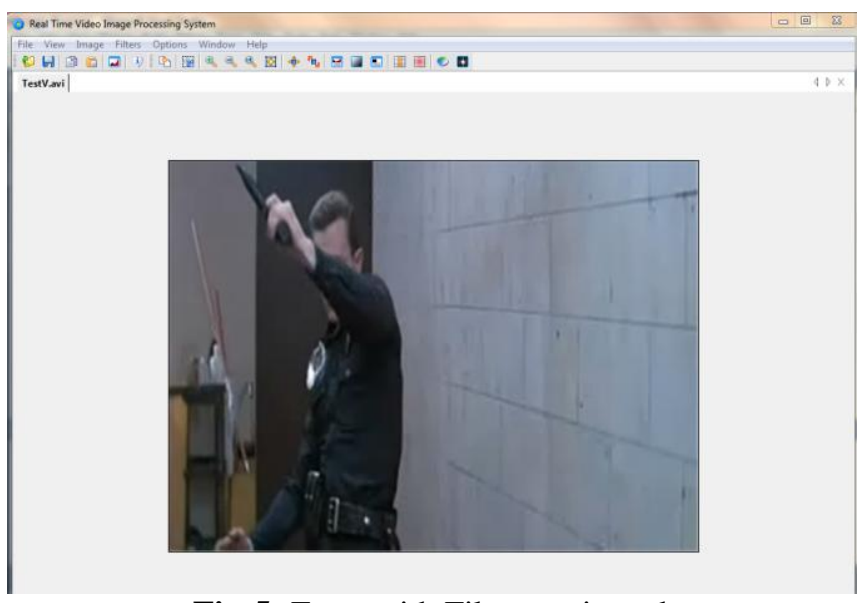

Fig-5: Form with Filters activated

3.3 Sobel Edge Detection Form: When the sobel edge dection filter is applied then the filter searches for objects edges. It filters out useless information and preserving the important structural properties in an image. The following figure 6 shows the output of the sobel edge detection form.

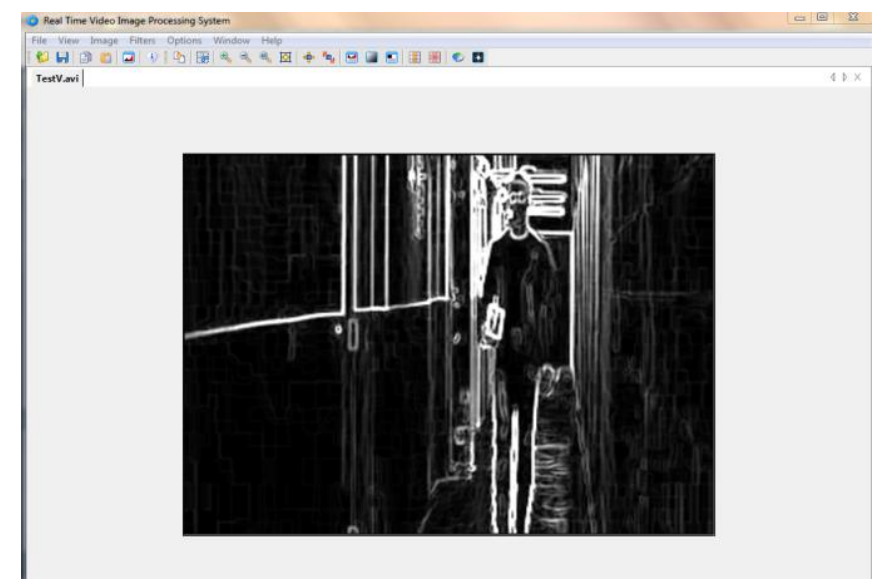

Fig-6: Applying the edge detection filter 
3.4 Brightness Form: The filter operates in HSL color space, and adjusts pixel's brightness value. Hear the brightness can be increased or decreased by specified percentage.

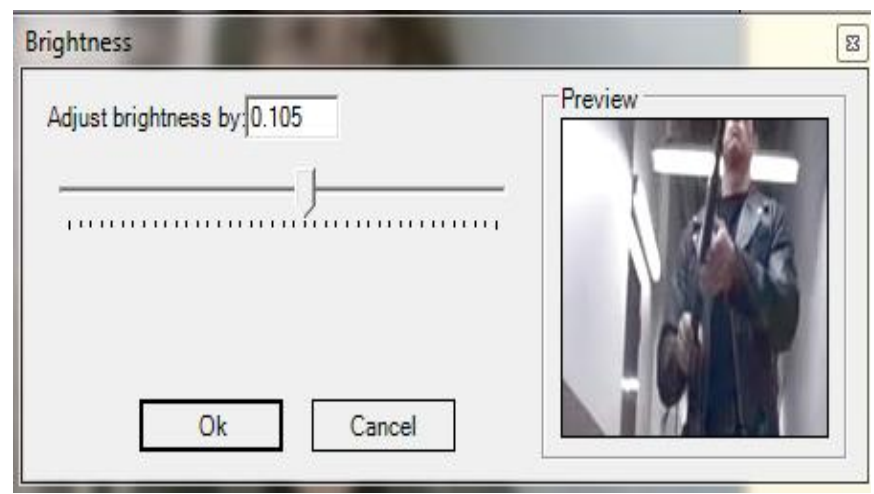

Fig-7: Brightness Form

\subsection{Perlin Noise Form:}

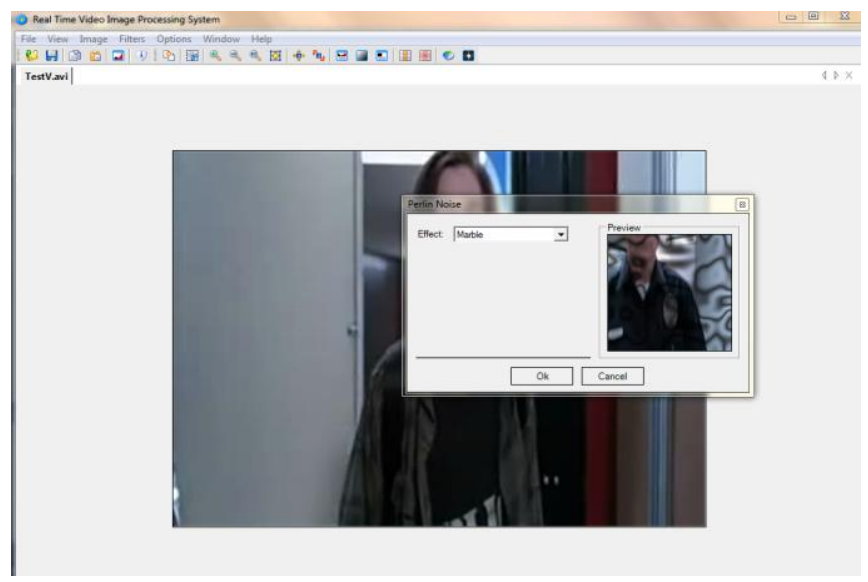

Fig-8: Perlin Noise Form

3.6 Color Filter Form: By applying color filters it keeps pixels with colors inside/outside of the specified sphere and fills the rest with specified color. The filter accepts 24 and 32 bpp color images for processing. The following figure 9 shows the gray scale image and 10 shows the extraction of red color image.

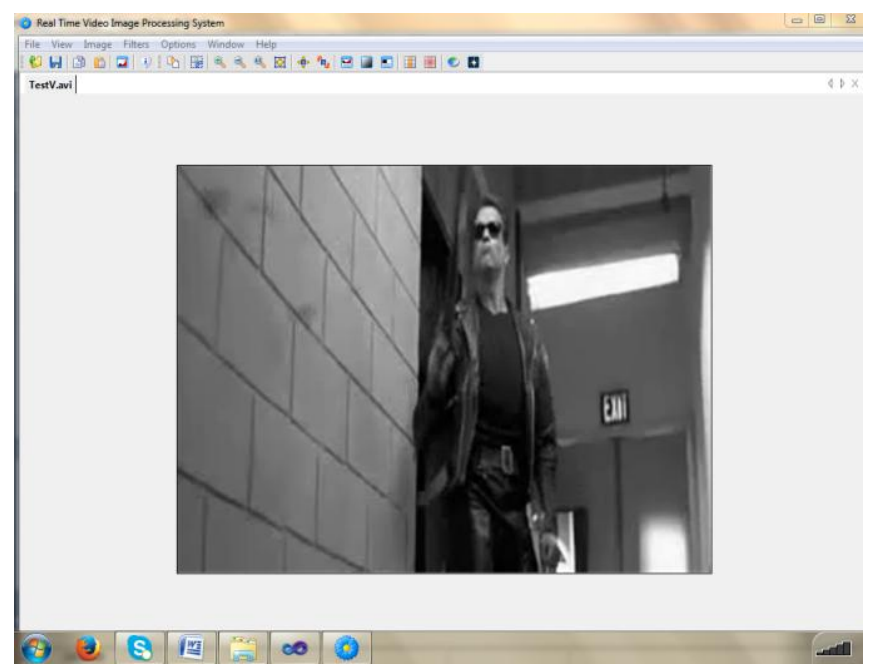

Fig-9: RGB image changed to Gray Scale image.

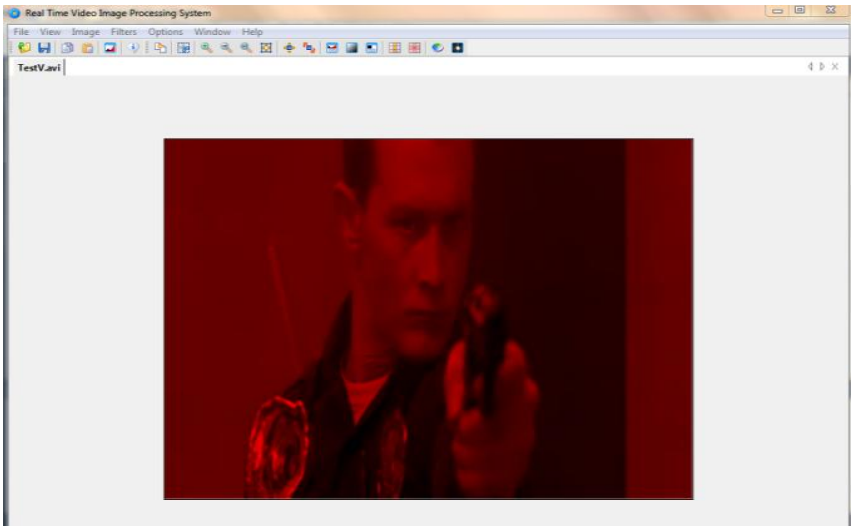

Fig-10: Extraction of Red Channel filter.

\section{CONCLUSION AND FUTURE SCOPE}

The video processing system is able to process the input video from the web camera. This system used for processing the video for enhancement, analysis, dividing the channels and binarization. Different filtering techniques are used for processing any type of video. By this video processing system we can improve the quality of the video. In future we can record, pause and save the video after applying the filter and we can also process the $3 \mathrm{D}$ videos in future.

\section{ACKNOWLEDGEMENT}

I am very thankful to the Dr.G.RameshChandra. I would like to acknowledge the cooperation of the management and staff of VNRVJIET.

\section{REFERENCES}

[1] Gonzalez, R.C. and R.E. Woods, Digital Image Processing, Addision-Wesley, 1992

[2] Lipton A, Fujiyoshi H, Patil R. Moving target classification and tracking from real-time video. In: proceedings IEEE image understanding workshop, 1998. 129-36

[3] Wren C,Azarbayejani A,Darrell T,Pentland Ap. Pfinder:realtime tracking of the human body. IEEE Transactions on pattern Analysis and Machine Intelligence 1997; 19(7):780-5.

[4] Haritaoglu I,Harwood D,Davis LS.W4:real-time surveillance of people and their activities. IEEE Transactions on pattern Analysis and MachineIntelligence 2000;22(8):809-30.

[5] J.C. Simon, O. Baret, N. Gorski, A system for the recognition of handwritten literal amounts of checks. In Proceedings of the Conference on Document Analysis system, Kaiserslautern, Germany, 1994, pp. 135-155

[6] H.G. Zhang, G. Chen, G. Liu, J. Guo, Bank check image

[7] binarization based on signal matching. In: Fifth International Conference on Information, Communications and Signal Processing, 2005, pp. 1430-1433. 
[8] M. Kamel, A. Zhao, Extraction of binary character/graphics images from grayscale document images, Graph. Models Image Process 55 (3) (1993) 203-217.

[9] Pitas I, Venetsanooulos AN. Nonlinear digital filters: principles and applications. Boston, MA: Kluwer; 1990.

[10] Astola J, Kuosmanen P. Fundamentals of nonlinear digital filtering. Boca Raton, FL: CRC Press; 1997.

[11] Machuca R, Phipps K. Applications of vector fields to image processing. IEEE Trans Pattern Anal Mach Intell 1983; PAMI- 5(3):316-29.

[12] W.K. Pratt, Digital Image Processing, Wiely, New York, 1978.

[13] How-Lung Eng, Kai-Kung Ma, Noise adaptive soft switching median filter, IEEE Trans. Image Process. 10 (2001) 1834-1838.

[14] S.-J. Ko, S.-J. Lee, Center weighted median filters and their applications to image enhancement, IEEE Trans. Circuits Syst. 15(1991) 984-993.

[15] Pitas, A. Venetsanopou, Nonlinear Digital Filters: Principles and Application, Kluwer, Norwell, MA, 1990.

\section{BIOGRAPHIES}

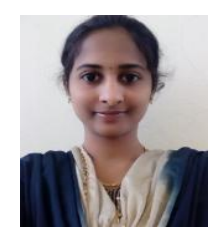

C.Keerthana P.G Student, Software Engineering, VNR Vignana Jyothi Institute of Engineering and Technology, Telangana, India

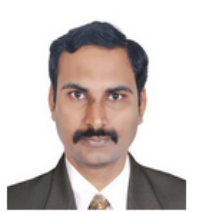

Dr. G. Ramesh Chandra, did his PhD in the area of image processing with thesis titled " Detection of Superficial and Volumetric Features in 3-D Digital Images" under JNTU-H. He is working as Professor in the Department of CSE. He has 15 Years of teaching and research experience. His areas of interest are Image processing, video processing and Data Mining. He is a Head of Research and Consultancy Centre (RCC) at VNR VJIET. He also developed a compiler for "Xervo Script Engine" for Cyber Motion Technologies Pvt. Ltd. He has published 23 research papers in international journals and conferences. 